\title{
Interkulturelle Germanistik reloaded - Pilotprojekt für NachwuchswissenschaftlerInnen
}

\author{
Bericht zum 1. Forum junger Wissenschaftlerlnnen der \\ Gesellschaft für interkulturelle Germanistik (GiG) \\ vom 10. bis 11. April 2019 an der Universität Zadar (Kroatien)
}

Isabelle Leitloff / René Perfölz/Fabian Wilhelmi

Im Juni 2018 verkündete die Gesellschaft für interkulturelle Germanistik (GiG) die Planung des ersten GiG-Forums junger WissenschaftlerInnen, das letztendlich sieben DoktorandInnen aus fünf Ländern in Zadar, Kroatien, zusammengebracht hat. Das GiG-Forum junger WissenschaftlerInnen wurde von den Mitgliedern des Vorstandes der GiG, Prof. Dr. Dieter Heimböckel von der Universität Luxemburg und Prof. Dr. Manfred Weinberg von der Karls-Universität Prag, initiiert, geleitet und durch Prof. Dr. Tomislav Zelić von der Universität Zadar unterstützt. GiG-Tagungen versammeln normalerweise knapp 180 WissenschaftlerInnen aus allen Teilen der Welt, davon sind jedoch viele ProfessorInnen und nur wenige DoktorandInnen. Bei den vielen parallelen Panels haben junge WissenschaftlerInnen zwar die Gelegenheiten, eigene Vorträge zu halten, jedoch bleiben bei Großveranstaltungen die Möglichkeiten begrenzt, dem wissenschaftlichen Nachwuchs einen eigenen Raum zu geben. Das neue Format der GiG soll daran etwas ändern. Das GiG-Forum soll Promotionsvorhaben wissenschaftlich unterstützen und das Gespräch zwischen den NachwuchswissenschaftlerInnen sowie ihre internationale Vernetzung fördern. Darüber hinaus bietet es die Möglichkeit, den Nachwuchs kennenzulernen: Wo stehen die jungen WissenschaftlerInnen? Was für Fragen beschäftigen sie und mit welchen Theorien und Methoden setzen sie sich auseinander?

Am 10. und 11. April kamen junge WissenschaftlerInnen aus Ägypten, Dänemark, Kroatien, Tschechien und Deutschland zusammen, stellten ihre Dissertationsthemen im Bereich der Interkulturalitätsforschung vor, nahmen an einer Meisterklasse zum Thema Mediterranismus teil und brachten sich aktiv in die Diskussion über zukünftige Veranstaltungsformate ein. Der Bericht über das GiG-Forum fasst dies im Folgenden zusammen und wirft einen Ausblick auf mögliche zukünftige Formate des jungen Pilotprojektes.

Mervat Abdelalim (Helwan Universität in Kairo, Ägypten) arbeitet als wissenschaftliche Mitarbeiterin an der Philosophischen Fakultät im Bereich der Germanistik. Ihre Forschungsschwerpunkte liegen auf dem Gebiet der Gender Studies sowie der Literatur des 20. und 21. Jahrhunderts. Diese Schwerpunkte 
sind ebenfalls in ihrer Dissertation vertreten, die sich mit dem sozialen Aufstieg marginalisierter weiblicher Figuren in Bildungsromanen der Gegenwart auseinandersetzt. Es werden sechs Romane zweier Autorinnen bezüglich der genannten Thematiken untersucht: Angelika Klüssendorfs Das Mädchen (2011), April (2014) und Jahre später (2018) sowie Emine Sevgi Özdamars Trilogie Sonne auf halbem Weg (2006). Anders als in bisherigen Arbeiten zum Bildungsroman soll dieser als process of self-development verstanden werden. Die ausgewählten Romane zeichnen ein Porträt des sozialen Aufstiegs und Erfolgs zweier Protagonistinnen, die am Rande der Gesellschaft leben und sich emanzipieren. Methodisch arbeitet Abdelalim mit Pierre Bourdieus soziologischem Ansatz des Habitus und untersucht die Einflüsse sozialer Räume auf den Habitus der weiblichen Hauptfiguren. Michel Foucaults Theorien der Macht und der Mechanismen der Exklusion dienen dazu, Marginalisierungsprinzipien sichtbar zu machen und den sozialen Stand der Protagonistinnen genauer bestimmen zu können. Die TeilnehmerInnen der Gruppendiskussion griffen den Terminus des Bildungsromans auf und erörterten die Frage, ob wir bei den oben genannten Romanen von Bildungsromanen sprechen können. Dabei wurde auf Ortrud Gutjahrs Einführung zum Bildungsroman verwiesen (vgl. Gutjahr 2007), in dem der weibliche, interkulturelle Bildungsroman ebenfalls vorgestellt wird. Auch die Ideen anderer TheoretikerInnen aus den Gender Studies wie Butler oder Spivak und konkret Spivaks Can the Subaltern Speak? wurden besonders in Bezug auf Özdamar und den Topos des »politischen Gegners« als Analysewerkzeug vorgeschlagen.

Dagmar Alešíková (Masaryk-Universität in Brünn, Tschechien) beschäftigt sich in ihrem Projekt mit Problemfeldern der Holocaustliteratur. Hierzu greift sie auf Vorarbeiten von Dan Diner (vgl. 2007) und Hayden White (vgl. 1986) zurück, die sich aus erkenntnistheoretischer und geschichtsphilosophischer Perspektive mit Fragekomplexen der Holocaustliteratur beschäftigt haben. Hieran anknüpfend fokussiert Alešíková auf die Publikationsflut von Holocaustzeitzeugnissen in den 1990er-Jahren und fragt, inwieweit diese Flut und die sich dadurch dynamisierenden erinnerungskulturellen Debatten innerhalb der Gesellschaft und der Forschung zu gegenwärtigen Bewertungen des Judentums beitrugen. Welche Rolle spielten die Publikationen im zwischenmenschlichen Austausch von Juden und Nichtjuden, und trägt die Rezeption der Zeitzeugnisse in der Gegenwart zu einer Eindämmung von antisemitischen, xenophoben sowie rassistischen Vorstellungen in der deutschen Gesellschaft bei? Um diese Fragen zu beantworten, analysiert Alešíková die autobiographischen und kritischen Schriften von Ruth Klüger und Marcel Reich-Ranicki unter der Fragestellung, in welcher Form die untersuchten Texte das Holocaustgeschehen re-konfigurieren und wie sie die Erinnerungskultur beeinflusst haben. Zentral ist dabei die Analyse von Klügers Weiter leben. Eine Jugend (1992) und Reich-Ranickis Mein Leben (1999). Alešíková wählt für ihre Untersuchung einen literaturwissenschaftlichen Zugang, der sich methodisch an Stephan Braeses und Holger Gehles Literaturwissenschaft und Literaturgeschichte nach dem Holocaust (Braes/Gehle 1999) sowie Hermann Kortes Untersuchung Der Holocaust in der Lyrik nach 1945 (Korte 1999) orientiert. 
Isabelle Leitloff ist Doktorandin und Lehrbeauftragte der Universität Paderborn und forscht im Bereich der interkulturellen Literatur- und Kulturwissenschaft. In ihrer Dissertation mit dem (Arbeits-)Titel Transatlantische Räume, plurale Identitäten: >Race < und Gender in der kubanischen und brasilianischen Kultur aus der interkulturellen Perspektive literarischer Texte Georgina Herreras, Nancy Morejóns, Soleida Ríos, Lydia Cabreras, Mãe Beata de Yemonjás und Hubert Fichtes steht Literatur im Fokus, die über reine Ursprünge, lineare Strukturen und über ein Denken in dualistisch-binären Kategorien hinausgeht. Die ausgewählten Werke, so die These, verstehen postkoloniale Räume nicht mehr nur als Widerstandsräume, sondern als hochpotenzierte interkulturelle Räume, die Kontinente miteinander verbinden und andere sprachliche, literarische und philosophische Prozesse anregen. Deshalb werden der Prozess der Identifikation, die Frage nach Genderidentitäten und Synkretismen in der brasilianischen und kubanischen Literatur in den Werken analysiert. In Herreras und Morejóns Werken wird auf polare und duale Codes aufmerksam gemacht, und sie werden hinterfragt. Letzteres sei ebenfalls in Fichtes Explosion. Roman der Ethnologie (1993) der Fall. Anhand der doppeltblickenden Methode, die durch den Vergleich brasilianischer Literatur von Mãe Beata de Yemonjá mit der von Fichte zustande kommt, soll das Fremdverstehen aufgegriffen und der Frage nachgegangen werden, ob doppeltblickende Verfahren nach Leo Kreutzer (vgl. 2009) einen Mehrwert erbringen können, ohne in eurozentrische Muster zu verfallen. Methodisch arbeitet Leitloff nicht nur mit bekannten Theorien wie der Homi K. Bhabhas, sondern sie arbeitet Kreutzers Theorie des Doppelblicks aus und bringt Fernando Ortiz' Theorien (vgl. Ortiz 1978) in die interkulturelle Germanistik ein. In der anschließenden Diskussion stand vor allem der Begriff des Transatlantiks im Mittelpunkt.

Katrine Fleckner Gravholt (Europa-Universität Flensburg) schreibt ihr Dissertationsprojekt im Fachbereich der transkulturellen Pädagogik. Die Doktorandin interessiert sich für die didaktischen und pädagogischen Rahmenbedingungen, welche eine produktive Zusammenarbeit zwischen deutschen und dänischen SchülerInnen in der Auseinandersetzung mit Lerngegenständen an außerschulischen Lernorten fördern. Sie geht davon aus, dass die SchülerInnen durch transkulturelle Verflechtungen zwischen den Ländern viel kulturelles Wissen teilen. Die Zusammenarbeit sollte dementsprechend vor allem von lern- oder motivationstheoretischen Faktoren abhängen. Als Analysemethode verwendet Fleckner Gravholt die teilnehmende Beobachtung während mehrerer Unterrichtseinheiten in deutschen und dänischen Museen und anschließende Befragungen. Erste Befunde zeigen, dass die Zusammenarbeit durch sprachliche Hürden und durch eine unpassende Aufbereitung der Lerngegenstände erschwert ist.

Die anschließende Diskussion beschäftigte sich u.a. mit der Frage, ob der Begriff der Kultur als sinnvoll für die geplanten Analysen erscheint. Demgegenüber spezifizierte Fleckner Gravholt ihren Begriff als soziale Praxis innerhalb des organisatorischen und didaktischen Rahmens in den Museen. Um die Ergebnisse der Arbeit für die Museumsdidaktik nutzbar zu machen, wurde ein Kon- 
zept von interkulturellem Staunen in die Diskussion eingebracht (vgl. Heimböckel/Weinberg 2014): Das Staunen über das vermeintlich Andere könne zu einer intensiveren Auseinandersetzung mit gemeinsamen Lerngegenständen führen.

René Perfölz (Humboldt-Universität, Berlin) untersucht in seinem Dissertationsprojekt Inszenierungsformen und Darstellungsweisen von Zugehörigkeit in der türkisch-deutschen (Post-)Migrationsliteratur, um darzulegen, wie sich >türkische< Figuren seit der Wiedervereinigung in der deutschen Kultur und Gesellschaft verorten. Bei der Darstellung von Zugehörigkeit in den Texten spielten gerade Räume eine wichtige Rolle. Perfölz unterscheidet verschiedene Dimensionen räumlicher Zugehörigkeit: Resonanz, Raumaneignung durch re-mapping oder hybride Grenzzonen sowie Entortung durch Ausgrenzung oder Entfremdung. Für die Bestimmung der Dimensionen analysiert er sein Forschungskorpus mit einem postklassischen (Raum-)Narratologie-Modell. Dabei gelte es, verschiedene Aspekte des narrativen Raums zu untersuchen und aufeinander zu beziehen sowie Raumtypen wie Nicht-Orte oder Erinnerungsorte auf ihre Funktion in den jeweiligen Texten hin zu befragen. Der Untersuchungsfokus liegt auf dem kulturell präfigurierten Raum Berlin.

In der Diskussion wurde unterschieden zwischen einem Heterotopos (Foucault) und einem Nicht-Ort (Augé). Dabei zeigte sich, dass Raumtypen nicht schematisch bestimmten Orten zugewiesen werden können, sondern in Relation zu anderen Räumen und unter Einbeziehung der narrativen Darstellung bestimmt werden müssen. Ein weiterer Diskussionspunkt war die Gattungsbezeichnung Migrationsliteratur. Gegenüber dem Begriff der interkulturellen Literatur sah Perfölz seine Gattungsbezeichnung insofern als angemessen an, als die zu untersuchenden Texte Prozesse transnationaler Migration und damit zusammenhängende Themen wie Kulturalisierung, Fremd- und Selbstbestimmung sowie Diskriminierung inszenieren würden.

Kristijan Tomasović' (Universität Zadar) Promotionsprojekt fokussiert die Frage, in welcher Weise Literatur auf die zunehmende Säkularisierung innerhalb der Gesellschaft reagiert. Zur Analyse herangezogen werden Werke wie Maxim Billers Die Tochter (2000) und Thomas Pynchons Gravity's Rainbow (1973). Im Vordergrund steht aber das Werk W.G. Sebalds. Von besonderem Interesse sind dabei Sebalds postmoderne Reiseberichte. Die Protagonisten der untersuchten Texte deutet Tomasović als Beispiele des postsäkularen Menschen: Die eigentliche Reise sei ihnen weniger wichtig. Im Vordergrund stünde vielmehr der pluralistische Individualismus, die Sinnsuche und Selbsterfüllung, die eine neue Form der Religiosität darstelle. Tomasović stützt sich bei seiner Analyse auch auf empirische Befunde, denen zufolge es gerade bei jungen Menschen zu einer Entzweiung von Religion und Religiosität komme. Ebenso eine Rolle spielen die Ansätze von David Jasper (vgl. 2009) und Wolfgang Frühwald (vgl. 2008), die das Streben nach Religiosität im postsäkularen Zeitalter aus literaturwissenschaftlicher Perspektive beleuchtet haben. Tomasović' erste Analyseergebnisse zeigten, dass die Protagonisten in den untersuchten Texten Sebalds ähnlich auf die Säkularisierung reagieren. So lasse sich nachweisen, dass neue, 
individuelle sakrale Formen bei der Sinnsuche der Hauptfiguren eine große Rolle spielen.

Den Abschluss der Projektvorstellungen bildete Fabian Wilhelmis (Universität Düsseldorf) Dissertationsvorhaben, das den Arbeitstitel Literarischer Antisemitismus in historischen Romanen über den Jüdischen Krieg aus dem späten 19. und frühen 20. Jahrhundert trägt. Wilhelmis Ansatz besteht darin, die Auswirkungen des sich im 19. Jahrhundert vollziehenden Wandels von Antijudaismus zu Antisemitismus in historischen Romanen über den Jüdischen Krieg (66-70 n.Chr.) zu untersuchen. Anhand der Gegenüberstellung der im 19. Jahrhundert erschienenen Texte und jenen, die im 20. Jahrhundert veröffentlicht wurden, will Wilhelmi zeigen, dass das Ausmaß antisemitischer Elemente in den später erschienenen Texten einen höheren Anteil einnimmt. Dadurch könne belegt werden, dass der virulenter werdende Antisemitismusdiskurs durch interdiskursive Praktiken auch Auswirkungen auf die literarische Produktion gehabt habe. Wilhelmi zieht zur Stützung seiner These auch außerliterarische Texte wie Zeitungsartikel, Rezensionen, biographische Dokumente und historiographische Texte heran. Erste Ergebnisse Wilhelmis bestätigen, dass die Figuren in den untersuchten Romanen nach ähnlichen Schemata konzipiert worden sind. Für den weiteren Fortgang der Studie ist die Suche nach gegenläufigen Figurendarstellungen, die nicht das zeitgenössische Stereotyp widerspiegeln, von besonderem Interesse.

Für den Vormittag des zweiten Programmtages war eine Meisterklasse von Tomislav Zelić von der Universität Zadar zum Konzept des Mediterranismus eingeplant. Der Begriff bezieht sich auf literarische und kulturelle Imaginationen des Mittelmeers und des europäischen Südens. Seine Geschichte spannt sich von Ovids Erzählung des Raubs Europas durch den griechischen Gott Zeus über die Grand Tour des 18. Jahrhunderts bis hin zum Noucentisme, einer katalanischen Form des Neoklassizismus. Zelić verglich das Konzept mit Raumkonzepten wie dem Orientalismus oder dem Balkanismus: Es handle sich um eine europäische Diskursformation, die dem durch den Begriff des Mittelmeers bezeichneten Raum bestimmte Eigenschaften zuweise und ihn dadurch homogenisiere. Der Mittelmeerraum nehme als >anderer Raum eine identitätsbildende Funktion für Europa ein und versichere es seiner eigenen kulturellen und intellektuellen Überlegenheit. Im Anschluss an die Vorstellung des Konzepts wurde dessen Anwendung im Rahmen eines Werkstattseminars erprobt.

In der folgenden Diskussion zeigten sich z.T. Schwierigkeiten, den Begriff des Mediterranismus von Raumkonzepten wie dem Orientalismus abzugrenzen. Als mögliche Gefahr des Ansatzes wurde der Fokus auf binäre Raumkonstruktionen benannt, der zu (Re-)Essentialisierungen und zur Vernachlässigung von raum-zeitlichen Ambivalenzen führen könne. Kritisch bemerkt wurde auch die eurozentrische Perspektive des Konzepts, das vornehmlich den Blick (nordund west-)europäischer AutorInnen auf den europäischen Süden untersuche. Zuletzt wurde auch der Bezugsraum des Konzepts von den TeilnehmerInnen diskutiert, da die exemplarischen literarischen Beispieltexte mit Italien und Griechenland lediglich Topographien der Klassik inszenierten. 
Als Fazit des Pilotprojektes für NachwuchswissenschaftlerInnen und als Ausblick soll Folgendes festgehalten werden: Forschungen im Bereich der Interkulturalität waren, sind und werden ein wichtiger Bestandteil der Germanistik sein. Besonders in Hinblick auf globale Nationalisierungstendenzen zeigt sich, dass auch die Wissenschaft und besonders die Germanistik gefragt sind, interkulturelle Forschungen zu fördern, nationale Grenzen zu überschreiten und überregionale und interkulturelle Forschungsnetzwerke zu erhalten und aufzubauen. Deshalb ist die Förderung junger WissenschaftlerInnen, die sich in den Bereichen der Interkulturalität bewegen, unabdingbar.

Die interkulturelle Germanistik wächst besonders durch interkulturelle Begegnungen, die nicht nur in literarischen Werken verortet werden. Um Interkulturalität zu erforschen, ist ein Zusammenkommen, ein gemeinsames Aushandeln, Verhandeln, Diskutieren und Debattieren wichtig, um die zukünftige Wissenschaftslandschaft mitgestalten zu können und Interkulturalität nicht nur zu theoretisieren. Als Ausblick für zukünftige Formate wurden im Rahmen der Abschlussdiskussion Ideen gesammelt: Es könnten vorab Ausschnitte einiger Primär- und Sekundärtexte eingereicht werden, die einen tieferen Einstieg in die Thematik ermöglichen. Außerdem wurde das Format des Workshops als alternative Präsentationsform vorgeschlagen, die Meisterklasse wurde gelobt und ist weiterhin erwünscht.

Für das Ermöglichen der interkulturellen Begegnung möchten wir uns sehr herzlich im Sinne aller TeilnehmerInnen bei der GiG und den Veranstaltern des Forums bedanken. Die GiG hat mit dem neuen Konzept einen Impuls gegeben, der aufgenommen werden soll: Das GiG-Forum junger WissenschaftlerInnen soll zukünftig regelmäßig mit divergenten fachlichen Schwerpunkten angeboten werden und somit auch anderem wissenschaftlichen Nachwuchs die Möglichkeit einer interkulturellen Mitgestaltung der Wissenschaft geben. In diesem Sinne: Hvala und doviđenja.

\section{LITERATUR}

Braese, Stephan/Gehle, Holger (1999): Literaturwissenschaft und Literaturgeschichte nach dem Holocaust. In: Literatur und Holocaust. Text+Kritik. Zeitschrift für Literatur, H. 144, S. 79-95.

Diner, Dan (2007): Gegenläufige Gedächtnisse. Über Geltung und Wirkung des Holocaust. Göttingen.

Frühwald, Wolfgang (2008): Das Gedächtnis der Frömmigkeit: Religion, Kirche und Literatur in Deutschland. Vom Barock bis zur Gegenwart. Frankfurt a.M.

Gutjahr, Ortrud (2007): Einführung in den Bildungsroman. Darmstadt.

Heimböckel, Dieter/Weinberg, Manfred (2014): Interkulturalität als Projekt. In: Zeitschrift für interkulturelle Germanistik 5, H. 2, S. 119-144.

Jasper, David (2009): The Sacred Body: Asceticism in Religion, Literature, Art, and Culture. Waco. 
Korte, Hermann (1999): „Es ist in aller Trauer der tiefste Hang zur Sprachlosigkeit». Der Holocaust in der Lyrik nach 1945. In: Literatur und Holocaust. Text+Kritik. Zeitschrift für Literatur, H. 144, S. 25-47.

Kreutzer, Leo (2009): Goethe in Afrika. Die interkulturelle Literaturwissenschaft der "École de Hanovre" in der afrikanischen Germanistik. Hannover.

Ortiz, Fernando (1978): Contrapunteo cubano del tabaco y el azúcar. Caracas.

White, Hayden V. (1986): Auch Klio dichtet oder die Fiktion des Faktischen. Studien zur Topologie des historischen Diskurses. Aus dem Amerik. v. Brigitte BrinkmannSiepmann u. Thomas Siepmann. Stuttgart. 
